\title{
Periodic solutions for systems of coupled nonlinear Schrödinger equations with five and six components
}

\author{
K. W. Chow* and D. W. C. Lai \\ Department of Mechanical Engineering, University of Hong Kong, Pokfulam Road, Hong Kong \\ (Received 4 June 2001; revised manuscript received 18 September 2001; published 25 January 2002)
}

\begin{abstract}
Systems of coupled nonlinear Schrödinger (CNLS) equations arise in several branches of physics, e.g., optics and plasma physics. Systems with two or three components have been studied intensively. Recently periodic solutions for CNLS systems with four components are derived. The present work extends the search of periodic solutions for CNLS systems to those with five and six components. The Hirota bilinear method, theta and elliptic functions are employed in the process. The long wave limit is studied, and known results of solitary waves are recovered. The validity of these periodic solutions is verified independently by direct differentiation with computer algebra software.
\end{abstract}

DOI: 10.1103/PhysRevE.65.026613

PACS number(s): 42.65.Tg, 42.81.Dp, 02.30.Jr

\section{INTRODUCTION}

Systems of coupled nonlinear Schrödinger (CNLS) equations occur frequently in theory as well as in applications. The integrability and solitary waves of such CNLS systems have been studied intensively. The focus of the present work is on the periodic waves of these CNLS systems. More precisely, we shall study the CNLS system of $M$ components,

$$
i \frac{\partial \phi_{m}}{\partial t}+\frac{\partial^{2} \phi_{m}}{\partial x^{2}}+\left(\sum_{r=1}^{M} \phi_{r} \phi_{r}^{*}\right) \phi_{m}=0, \quad m=1,2,3, \ldots, M .
$$

An application of intensive recent interest is the use of CNLS and related systems as models for the propagation of optical solitons along fibers. Analytically a class of periodic waves for CNLS and related systems has been expressed as products of Jacobi elliptic functions [1-6]. These solutions are mainly for the case of two components, but some special solutions for three or four components have also been found.

A general algorithm based on an ansatz of Lamé functions has been developed. Properties of the periodic waves, e.g., the amplitude and the frequency, are solved as solutions of a system of algebraic equations. Reductions to the Jacobi elliptic functions for the case of $M=2,3$ are documented explicitly [7-9].

Solitons can propagate along an optical fiber by a balance of group velocity dispersion and self-phase modulation. To increase the information carrying capacity it will be desirable to propagate two or more fields simultaneously. A system of CNLS will then be a relevant model. The eigenvalue problem and the Bäcklund transformation can be investigated and one-soliton expressions can be derived explicitly [10]. The stability of multicomponent solitary waves can be studied [11].

Another area where the CNLS model will be applicable is the propagation of multimode, incoherent spatial solitons in noninstantaneous Kerr media. Optical spatial soliton can oc-

*FAX: (852) 2858 5415. Email address: kwchow@hkusua.hku.hk cur when diffraction and light-induced waveguiding effects are in balance. Recently incoherent spatial solitons were observed in noninstantaneous nonlinear media like biased photorefractive crystals. Two theories used to describe the phenomena are the coherent density method and the selfconsistent multimode method. In the second approach the total intensity of the multimode soliton solutions is the superposition of all the relevant modes in the nonlinear induced waveguide. Analytically the governing equation for each mode consists of a nonlinear Schrödinger-like operator, except the self-phase modulation nonlinearity is replaced by a linear term, with the total intensity as the multiplicative factor [12].

The Hirota bilinear operator and theta functions will be employed in the present paper. They have been demonstrated to be effective in treating solitary and periodic waves in the field of nonlinear waves. Indeed closed form solutions can be obtained when the total intensity profile of these incoherent solitons states is equal to the square of the hyperbolic secant [12]. Here analytical expressions are presented when the profile is equal to the square of a Jacobi elliptic dn function.

Self-trapping for these biased photorefractive crystals also admits partially coherent solitons on a finite background [13]. Mathematically the field is governed by a CNLS system of $M$ components. Stationary solutions with nonzero boundary conditions can be derived by a direct method, as the inverse scattering technique is not applicable due to the nonzero conditions in the far field. Physically at least one component will have a nonzero asymptotic value of the index change induced by the partially coherent solitons.

The objective of this paper is to study periodic solutions of CNLS systems of five and six components. From a careful examination of the cases of two, three, and four components, periodic solutions for CNLS of five and six components are deduced. The number of local maxima within each period will in general increase with the number of components for such CNLS systems. Increasing the number of interacting fields thus may facilitate the propagation of coupled periodic waves $[8,9]$.

\section{THE HIROTA BILINEAR METHOD}

The goal here is to seek special solutions of the CNLS systems by first using the bilinear forms: 


$$
\begin{gathered}
\phi_{m}(x, t)=\frac{g_{m}(x) \exp \left(-i \Omega_{m} t\right)}{f(x)} \text { for } f \text { real, } \\
f\left[D_{x}^{2} g_{m} \cdot f+\Omega_{m} g_{m} f\right]+g_{m}\left[-D_{x}^{2} f \cdot f+\sum_{r=1}^{M} g_{r} g_{r}^{*}\right]=0,
\end{gathered}
$$

where $D$ is the Hirota operator. The crucial difference between the present situation and the case of solitary waves is that the bilinear form (2.2) must now be used as a single equation, and not as two decoupled equations. We shall assume $g_{m}$ to be real here as well.

Second we choose,

$$
f=\left[\theta_{4}(\alpha x)\right]^{p}
$$

where $p$ is a small positive integer ( 5 or 6 in this paper). Formulas for the theta functions are found in the literature [14-16] and in the Appendix.

Finally $g_{m}$ must be chosen such that Eq. (2.2) is satisfied. The Hirota derivatives of theta functions are handled by theta identities (the Appendix). The critical step in formulating the correct form of $g_{m}$ is that sufficient powers of $\theta_{3}(x)$ must be canceled in Eq. (2.2) for the matching to be performed.

By invoking products of two and three theta functions, periodic solutions for CNLS equations with $M=2,3$ involving two and three elliptic or theta functions can be deduced or recovered. Recently the consideration is extended to CNLS equations of four components. A little experimentation shows that the arrangements

$$
\begin{aligned}
& g_{m}=A\left[c \theta_{4}^{2}(\alpha x)-\theta_{3}^{2}(\alpha x)\right] \theta_{1}(\alpha x) \theta_{2}(\alpha x) \exp (-i \Omega t), \\
& g_{m}=A\left[c \theta_{4}^{2}(\alpha x)-\theta_{3}^{2}(\alpha x)\right] \theta_{1}(\alpha x) \theta_{3}(\alpha x) \exp (-i \Omega t), \\
& g_{m}=A\left[c \theta_{4}^{2}(\alpha x)-\theta_{3}^{2}(\alpha x)\right] \theta_{2}(\alpha x) \theta_{3}(\alpha x) \exp (-i \Omega t),
\end{aligned}
$$

are possible candidates for solving Eqs. (2.2) [17]. Four linearly independent solutions are obtained by suitably choosing $A$ and $c$. A crucial step in the choice of $g_{m}$ and $A$ is that the sum of intensity, which is related to the "refractive index" $[12,18]$, is

$$
\sum_{r=1}^{4} \phi_{r} \phi_{r}^{*}=h_{0}+h_{1}\left(\frac{\theta_{3}^{2}(\alpha x)}{\theta_{4}^{2}(\alpha x)}\right)=h_{0}+\frac{h_{1} \mathrm{dn}^{2}(r x)}{\left(1-k^{2}\right)^{1 / 2}},
$$

where $h_{0}$ and $h_{1}$ are constants.

The main goal of the present work is to present special periodic solutions for CNLS systems with five and six components. The solutions are deduced from special cases for a more elaborate general theory outlined earlier in the literature. The case for $M=2$ is reported by Kostov and Uzunov [3], while the general integer $M$ case is documented by Hioe $[7]$.

\section{FORMS OF THE PERIODIC SOLUTIONS}

For CNLS systems of odd order, say, $M=2 N+1$, one possible reduction of the general theory is that, for each component, $m=1,2, \ldots, M$,

$$
\begin{aligned}
\phi_{m}= & A_{m}\left[a_{m, N} \theta_{4}^{2 N}(\alpha x)+a_{m, N-1} \theta_{4}^{2 N-2}(\alpha x) \theta_{3}^{2}(\alpha x)+\cdots\right. \\
& \left.+a_{m, 0} \theta_{3}^{2 N}(\alpha x)\right] F(x) \exp \left(-i \Omega_{m} t\right) / \theta_{4}^{2 N+1}(\alpha x), \\
\phi_{m}= & A_{m}\left[\xi_{m, N-1} \theta_{4}^{2 N-2}(\alpha x)+\xi_{m, N-2} \theta_{4}^{2 N-4}(\alpha x) \theta_{3}^{2}(\alpha x)\right. \\
& \left.+\cdots+\xi_{m, 0} \theta_{3}^{2 N-2}(\alpha x)\right] \theta_{1}(\alpha x) \theta_{2}(\alpha x) \theta_{3}(\alpha x) \\
& \times \exp \left(-i \Omega_{m} t\right) / \theta_{4}^{2 N+1}(\alpha x),
\end{aligned}
$$

where $F(x)$ will be taken from one of three choices: $\theta_{1}(\alpha x)$, $\theta_{2}(\alpha x), \theta_{3}(\alpha x)$. The coefficient $a_{m, N}$ will be determined by a polynomial of degree $N+1$. The remaining coefficient, $a_{m, r}, r=N-1, N-2, \ldots, 0$, will be related to $a_{m, N}$ by welldefined algebraic relations. $\xi_{m, N-1}$ will be determined by a polynomial of degree $N$ and $\xi_{m, r}, r=N-2, N-3, \ldots, 0$, are related to $\xi_{m, N-1}$ algebraically. The amplitude parameters $A_{m}, m=1,2, \ldots, M$, must be chosen such that

$$
\sum_{r=1}^{M} \phi_{r} \phi_{r}^{*}=h_{0}+h_{1}\left(\frac{\theta_{3}(\alpha x)}{\theta_{4}(\alpha x)}\right)^{2}
$$

where $h_{0}$ and $h_{1}$ are constants. In other words, the vanishing of $\left[\theta_{3}(\alpha x) / \theta_{4}(\alpha x)\right]^{2 r}, r=2, \ldots, M$, and a prescribed $h_{1}$ will define $M$ equations for $A_{m}^{2}$ and $h_{0}$ will determine the frequency parameter $\Omega_{m}$. The resulting equations will be a linear system in $A_{m}^{2}$. The parameters must be chosen such that the solutions for each $A_{m}^{2}$ will assume a positive value.

For CNLS systems of even order, say, $M=2 N$, one possible form of periodic solutions might be

$$
\begin{aligned}
\phi_{m}= & A_{m}\left[b_{m, N-1} \theta_{4}^{2 N-2}(\alpha x)+b_{m, N-2} \theta_{4}^{2 N-4}(\alpha x) \theta_{3}^{2}(\alpha x)\right. \\
& +b_{m, N-3} \theta_{4}^{2 N-b}(\alpha x) \theta_{3}^{4}(\alpha x)+\cdots \\
& \left.+b_{m, 0} \theta_{3}^{2 N-2}(\alpha x)\right] G(x) \exp \left(-i \Omega_{m} t\right) / \theta_{4}^{2 N}(\alpha x),
\end{aligned}
$$

$m=1,2, \ldots, M . G(x)$ will be chosen from the three choices: $\theta_{3}(\alpha x) \theta_{1}(\alpha x), \theta_{3}(\alpha x) \theta_{2}(\alpha x)$, or $\theta_{1}(\alpha x) \theta_{2}(\alpha x)$. The coefficient $b_{m, N-1}$ will be determined by a polynomial of degree $N$. The remaining coefficients $b_{m, r}, \quad r=N-2, N$ $-3, \ldots, 0$ can again be expressed in terms of $b_{m, N-1}$. The constants $A_{m}$ need to satisfy the constraint (3.1) too.

All the intermediate calculations will be conducted using theta functions, as their Hirota derivatives can be handled by the huge variety of theta identities. Final results, however, will be reported in terms of Jacobi elliptic functions. Elliptic functions representations are more compact and more suitable for graphical treatment by software packages. Conversion formulas among the theta and elliptic functions are given in the Appendix. 


\section{CNLS EQUATIONS WITH FIVE COMPONENTS}

As an illustration consider CNLS of five $(M=5)$ components. The periodic solutions are ( $k$ is modulus of the Jacobi elliptic functions):

$$
\begin{gathered}
\phi_{1}=A_{1}\left[c_{1}-\frac{\mathrm{dn}^{2}(r x)}{\left(1-k^{2}\right)^{1 / 2}}\right] \\
\times \frac{k \operatorname{sn}(r x) \operatorname{cn}(r x) \mathrm{dn}(r x) \exp \left(-i \Omega_{1} t\right)}{\left(1-k^{2}\right)^{1 / 2}} \cdot \\
\phi_{2}=A_{2}\left[c_{2}-\frac{\delta_{2} \mathrm{dn}^{2}(r x)}{\left(1-k^{2}\right)^{1 / 2}}-\frac{\mathrm{dn}^{4}(r x)}{1-k^{2}}\right] \frac{\mathrm{dn}(r x) \exp \left(-i \Omega_{2} t\right)}{\left(1-k^{2}\right)^{1 / 4}}, \\
\phi_{3}=A_{3}\left[c_{3}-\frac{\delta_{3} \mathrm{dn}^{2}(r x)}{\left(1-k^{2}\right)^{1 / 2}}-\frac{\mathrm{dn}^{4}(r x)}{1-k^{2}}\right] \frac{\mathrm{dn}(r x) \exp \left(-i \Omega_{3} t\right)}{\left(1-k^{2}\right)^{1 / 4}}, \\
\phi_{4}=A_{4}\left[c_{4}-\frac{\delta_{4} \mathrm{dn}^{2}(r x)}{\left(1-k^{2}\right)^{1 / 2}}-\frac{\mathrm{dn}^{4}(r x)}{1-k^{2}}\right] \frac{\sqrt{k} \operatorname{on}(r x) \exp \left(-i \Omega_{4} t\right)}{\left(1-k^{2}\right)^{1 / 4}}, \\
\phi_{5}=A_{5}\left[c_{5}-\frac{\delta_{5} \mathrm{dn}^{2}(r x)}{\left(1-k^{2}\right)^{1 / 2}}-\frac{\mathrm{dn}^{4}(r x)}{1-k^{2}}\right] \sqrt{k} \operatorname{sn}(r x) \exp \left(-i \Omega_{5} t\right)
\end{gathered}
$$

$c_{n}, n=1$ is a root of

$$
3 c^{2}-2\left(\sqrt{1-k^{2}}+\frac{1}{\sqrt{1-k^{2}}}\right) c+1=0 .
$$

$\Omega_{n}, n=1$, is given by $\left[h_{0}\right.$ to be given later in Eq. (4.11)]

$$
\Omega_{n}+h_{0}=18 r^{2} c_{n} \sqrt{1-k^{2}}-16 r^{2}\left(2-k^{2}\right) .
$$

$c_{n}, n=2,3$ are roots of

$$
63 c^{3}+\left(99+\frac{24 k^{4}}{1-k^{2}}\right) c^{2}+\left(41+\frac{16 k^{4}}{1-k^{2}}\right) c+5=0 .
$$

$\delta_{n}$ and $\Omega_{n}, n=2,3$ are related by

$$
\begin{gathered}
\delta_{n}=4\left(\sqrt{1-k^{2}}+\frac{1}{\sqrt{1-k^{2}}}\right)\left(\frac{1}{c_{n}}-3\right)^{-1}, \\
\Omega_{n}+h_{0}=-18 r^{2} \delta_{n} \sqrt{1-k^{2}}-25 r^{2}\left(2-k^{2}\right) .
\end{gathered}
$$

$c_{n}, n=4$ is a root of

$$
\begin{aligned}
& 189 c^{3}+\frac{\left(219-171 k^{2}+24 k^{4}\right) c^{2}}{1-k^{2}}+\frac{\left(31-23 k^{2}+8 k^{4}\right) c}{1-k^{2}}+1 \\
& \quad=0 .
\end{aligned}
$$

$\delta_{n}$ and $\Omega_{n}, n=4$, are related by

$$
\delta_{n}=4\left(2 \sqrt{1-k^{2}}+\frac{3}{\sqrt{1-k^{2}}}\right)\left(\frac{1}{c_{n}}-9\right)^{-1},
$$

$$
\Omega_{n}+h_{0}=-18 r^{2} \delta_{n} \sqrt{1-k^{2}}-r^{2}\left(41-16 k^{2}\right) .
$$

$c_{n}, n=5$ is a root of

$$
\begin{aligned}
& 189 c^{3}+\frac{\left(219-267 k^{2}+72 k^{4}\right) c^{2}}{1-k^{2}}+\frac{\left(31-39 k^{2}+16 k^{4}\right) c}{1-k^{2}}+1 \\
& \quad=0 .
\end{aligned}
$$

$\delta_{n}$ and $\Omega_{n}, n=5$ are related by

$$
\begin{gathered}
\delta_{n}=4\left(3 \sqrt{1-k^{2}}+\frac{2}{\sqrt{1-k^{2}}}\right)\left(\frac{1}{c_{n}}-9\right)^{-1}, \\
\Omega_{n}+h_{0}=-18 r^{2} \delta_{n} \sqrt{1-k^{2}}-r^{2}\left(41-25 k^{2}\right) .
\end{gathered}
$$

$A_{n}, n=1,2,3,4,5$ satisfy

$$
-\frac{\sqrt{1-k^{2}} A_{1}^{2}}{k^{2}}+A_{2}^{2}+A_{3}^{2}+\frac{A_{4}^{2}}{k}-\frac{\sqrt{1-k^{2}} A_{5}^{2}}{k}=0,
$$

$$
\begin{aligned}
& \frac{\left(2-k^{2}+2 c_{1} \sqrt{1-k^{2}}\right) A_{1}^{2}}{k^{2}}+2 \delta_{2} A_{2}^{2}+2 \delta_{3} A_{3}^{2} \\
& +\left(\frac{2 \delta_{4}-\sqrt{1-k^{2}}}{k}\right) A_{4}^{2}+\left(\frac{1-2 \delta_{5} \sqrt{1-k^{2}}}{k}\right) A_{5}^{2}=0,
\end{aligned}
$$

$$
\begin{aligned}
& -\frac{\left[\left(1+c_{1}^{2}\right) \sqrt{1-k^{2}}+2 c_{1}\left(2-k^{2}\right)\right] A_{1}^{2}}{k^{2}}+\left(\delta_{2}^{2}-2 c_{2}\right) A_{2}^{2} \\
& \quad+\left(\delta_{3}^{2}-2 c_{3}\right) A_{3}^{2}+\left(\frac{\delta_{4}^{2}-2 c_{4}-2 \delta_{4} \sqrt{1-k^{2}}}{k}\right) A_{4}^{2} \\
& \quad+\left(\frac{2 \delta_{5}+\left(2 c_{5}-\delta_{5}^{2}\right) \sqrt{1-k^{2}}}{k}\right) A_{5}^{2} \\
& =0
\end{aligned}
$$

$$
\begin{gathered}
\frac{\left[c_{1}^{2}\left(2-k^{2}\right)+2 c_{1} \sqrt{1-k^{2}}\right] A_{1}^{2}}{k^{2}}-2 c_{2} \delta_{2} A_{2}^{2}-2 c_{3} \delta_{3} A_{3}^{2} \\
+\left(\frac{\left(2 c_{4}-\delta_{4}^{2}\right) \sqrt{1-k^{2}}-2 c_{4} \delta_{4}}{k}\right) A_{4}^{2} \\
+\left(\frac{\delta_{5}^{2}-2 c_{5}+2 c_{5} \delta_{5} \sqrt{1-k^{2}}}{k}\right) A_{5}^{2}=0 . \\
-\frac{c_{1}^{2} \sqrt{1-k^{2}} A_{1}^{2}+c_{2}^{2} A_{2}^{2}+c_{3}^{2} A_{3}^{2}+\frac{c_{4}\left(c_{4}+2 \delta_{4} \sqrt{1-k^{2}}\right) A_{4}^{2}}{k}}{k} k^{2} \\
-\frac{c_{5}\left(\sqrt{1-k^{2}} c_{5}+2 \delta_{5}\right) A_{5}^{2}}{k}=30 r^{2} \sqrt{1-k^{2}} .
\end{gathered}
$$

The constants $h_{0}$ and $h_{1}$ are given by 


$$
h_{0}=\frac{c_{5}^{2} A_{5}^{2}}{k}-\frac{c_{4}^{2} \sqrt{1-k^{2}} A_{4}^{2}}{k}, \quad h_{1}=30 r^{2} \sqrt{1-k^{2}} .
$$

Intensities of each component are shown in Figs. 1(a)-1(e) for typical values of $k$. Up to five local maxima per period are possible.

We verify by the software MATHEMATICA that Eqs. (4.1)(4.5) satisfy Eq.(1.1).

Long wave limit. The long wave limit $(k \rightarrow 1)$ is instructive as the solutions reduce to forms known in the literature. This limit thus provides an additional, independent confirmation on the validity of the periodic solutions here. It will be necessary to solve for $Y_{n}, n=1,2,3,4,5$, where

$$
\begin{gathered}
Y_{n}=\frac{A_{n}^{2}}{\left(1-k^{2}\right)^{5 / 2}} \quad \text { for } n=2,3,4, \\
Y_{n}=\frac{A_{n}^{2}}{\left(1-k^{2}\right)^{2}} \quad \text { for } n=1,5 .
\end{gathered}
$$

Equations (4.6)-(4.10) can be simplified considerably by taking the $k \rightarrow 1$ limit in the expressions for $c_{n}$ and $\delta_{n}$ given earlier in this section. The long wave limit of the Jacobi elliptic functions are $(\operatorname{sn}(r x), \operatorname{cn}(r x), \operatorname{dn}(r x))$ $\rightarrow(\tanh r x, \operatorname{sech} r x, \operatorname{sech} r x)$ as $k \rightarrow 1$.

After some algebraic manipulations we obtain

$$
\begin{gathered}
\phi_{1}=\frac{\sqrt{210} r S^{2} T\left(2-3 S^{2}\right) \exp \left(4 i r^{2} t\right)}{2}, \\
\phi_{2}=\frac{\sqrt{30} r S\left(8-28 S^{2}+21 S^{4}\right) \exp \left(i r^{2} t\right)}{8}, \\
\phi_{3}=\frac{15 \sqrt{7} r S^{5} \exp \left(25 i r^{2} t\right)}{8}, \\
\phi_{4}=\frac{3 \sqrt{35} r S^{3}\left(8-9 S^{2}\right) \exp \left(9 i r^{2} t\right)}{8}, \\
\phi_{5}=\frac{3 \sqrt{70} r S^{4} T \exp \left(16 i r^{2} t\right)}{2}, \\
S=\operatorname{sech} r x, \quad T=\tanh r x, \quad \sum_{m=1}^{5} \phi_{m} \phi_{m}^{*}=30 r^{2} S^{2} .
\end{gathered}
$$

This long wave limit thus generates solutions that agree with known results [12].

\section{CNLS EQUATIONS WITH SIX COMPONENTS}

As an example of CNLS systems of even order, consider $M=6$. For $n=1,2,3$, the components are ( $k$ is modulus of the Jacobi elliptic functions)

$$
\begin{aligned}
\phi_{n}= & A_{n}\left[c_{n}-\frac{\delta_{n} \mathrm{dn}^{2}(r x)}{\left(1-k^{2}\right)^{1 / 2}}-\frac{\mathrm{dn}^{4}(r x)}{1-k^{2}}\right] \\
& \times \frac{\sqrt{k} \operatorname{sn}(r x) \operatorname{dn}(r x) \exp \left(-i \Omega_{n} t\right)}{\left(1-k^{2}\right)^{1 / 4}} .
\end{aligned}
$$

$c_{n}, n=1,2,3$ are roots of

$$
\begin{aligned}
363 c^{3} & +\frac{11\left(47-55 k^{2}+16 k^{4}\right) c^{2}}{1-k^{2}}+\frac{\left(169-201 k^{2}+80 k^{4}\right) c}{1-k^{2}} \\
& +15=0 .
\end{aligned}
$$

$\delta_{n}, \Omega_{n}, n=1,2,3$ are related by

$$
\begin{gathered}
\delta_{n}=4\left(4 \sqrt{1-k^{2}}+\frac{3}{\sqrt{1-k^{2}}}\right)\left(\frac{3}{c_{n}}-11\right)^{-1}, \\
\Omega_{n}+h_{0}=-22 r^{2} \delta_{n} \sqrt{1-k^{2}}-r^{2}\left(61-36 k^{2}\right) .
\end{gathered}
$$

For $n=4,5,6$, the remaining components are

$$
\begin{aligned}
\phi_{n}= & A_{n}\left[c_{n}-\frac{\delta_{n} \operatorname{dn}^{2}(r x)}{\left(1-k^{2}\right)^{1 / 2}}-\frac{\operatorname{dn}^{4}(r x)}{1-k^{2}}\right] \\
& \times \frac{\sqrt{k} \operatorname{cn}(r x) \operatorname{dn}(r x) \exp \left(-i \Omega_{n} t\right)}{\left(1-k^{2}\right)^{1 / 2}} .
\end{aligned}
$$

$c_{n}, n=4,5,6$ are roots of

$$
\begin{aligned}
363 c^{3} & +\frac{11\left(47-39 k^{2}+8 k^{4}\right) c^{2}}{1-k^{2}}+\frac{\left(169-137 k^{2}+48 k^{4}\right) c}{1-k^{2}} \\
& +15=0 .
\end{aligned}
$$

$\delta_{n}, \Omega_{n}, n=4,5,6$ are related by

$$
\begin{gathered}
\delta_{n}=4\left(3 \sqrt{1-k^{2}}+\frac{4}{\sqrt{1-k^{2}}}\right)\left(\frac{3}{c_{n}}-11\right)^{-1}, \\
\Omega_{n}+h_{0}=-22 r^{2} \delta_{n} \sqrt{1-k^{2}}-r^{2}\left(61-25 k^{2}\right) .
\end{gathered}
$$

The constants $h_{0}, h_{1}$ are given by

$$
h_{0}=0, \quad h_{1}=42 r^{2} \sqrt{1-k^{2}} .
$$

$h_{0}$ is zero due to the special choices in Eqs. (5.1) and (5.2). If the combination $\operatorname{sn}(r x) \mathrm{cn}(r x)$ is included in addition to Eqs. (5.1) and (5.2), $h_{0}$ will be nonzero. $A_{n}, n=1,2, \ldots, 6$ satisfy

$$
\begin{gathered}
\sqrt{1-k^{2}}\left(\sum_{n=1}^{3} A_{n}^{2}\right)-\left(\sum_{n=4}^{6} A_{n}^{2}\right)=0, \\
\sum_{n=1}^{3}\left(1-2 \delta_{n} \sqrt{1-k^{2}}\right) A_{n}^{2}-\sum_{n=4}^{6}\left(\sqrt{1-k^{2}}-2 \delta_{n}\right) A_{n}^{2}=0,
\end{gathered}
$$



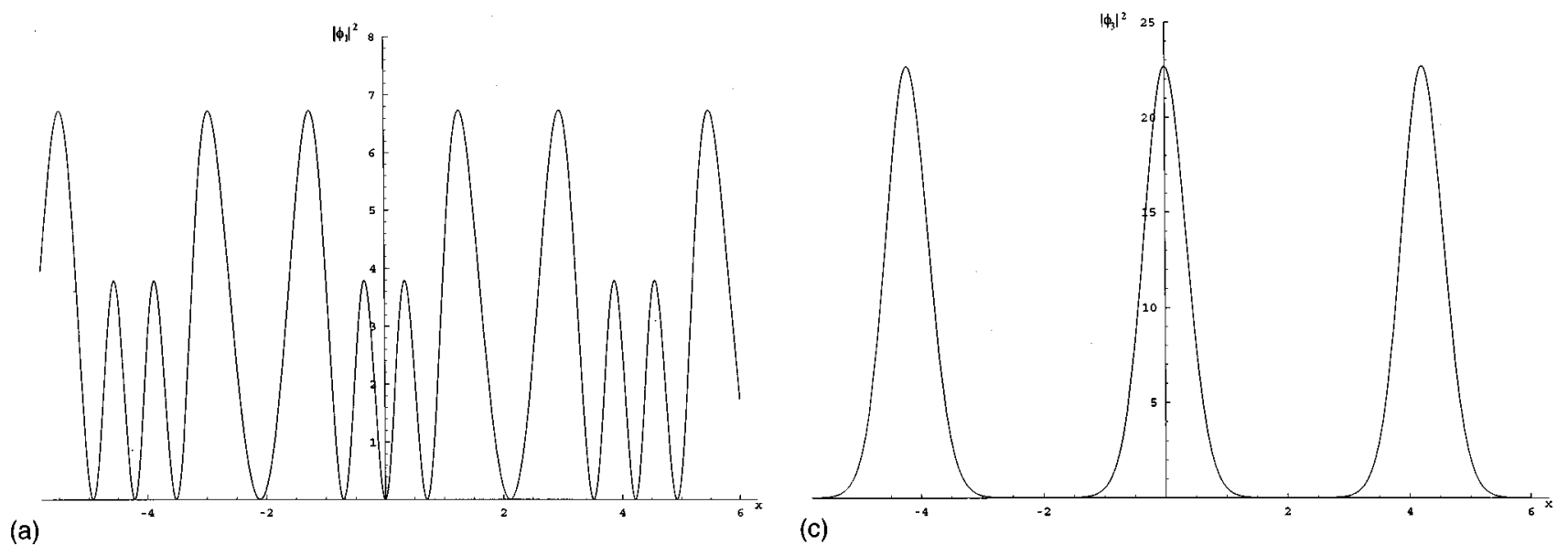

(a)

(c)
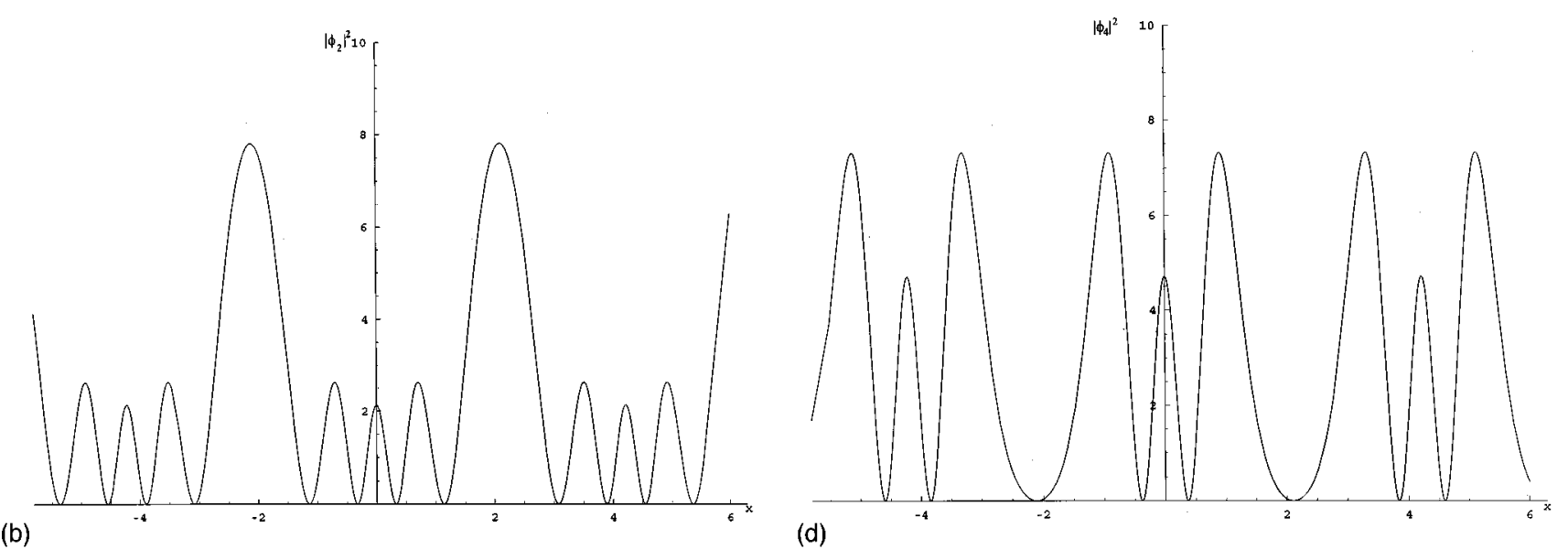

(d)

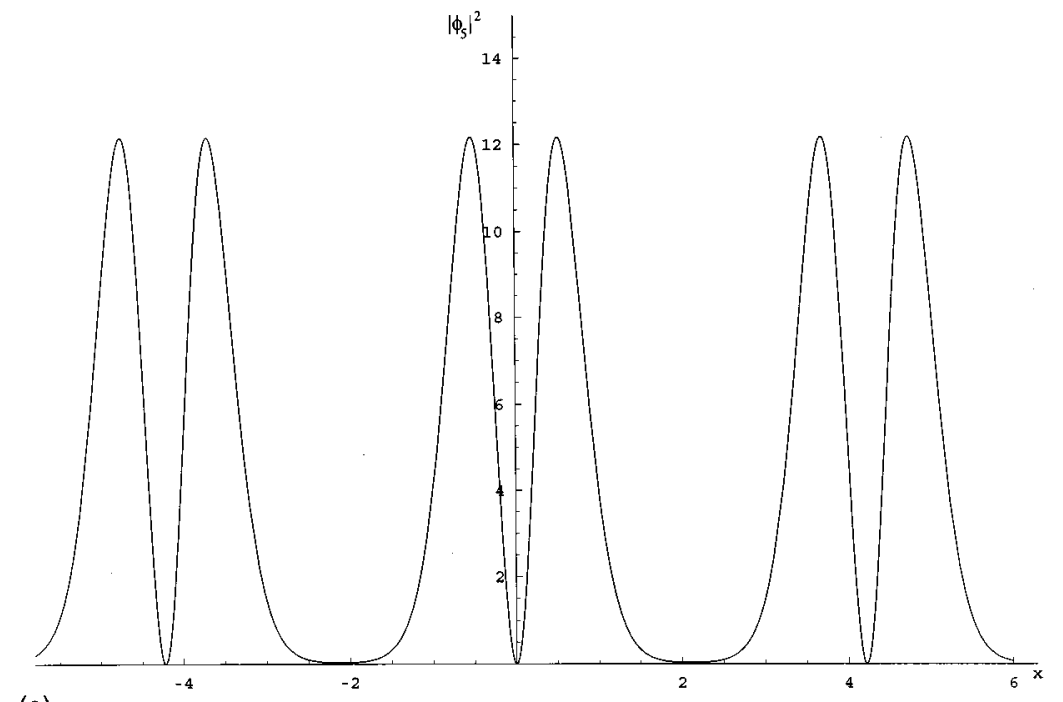

(e)

FIG. 1. (a) Plot of the nondimensional intensity $\left|\phi_{1}\right|^{2}$ versus the nondimensional coordinate $x, r=1, k=0.85, c_{1}=1.37, A_{1}=10.77$. (b) Plot of the nondimensional intensity $\left|\phi_{2}\right|^{2}$ versus the nondimensional coordinate $x, r=1, k=0.85, c_{2}=-1.62, A_{2}=7.90$. (c) Plot of the nondimensional intensity $\left|\phi_{3}\right|^{2}$ versus the nondimensional coordinate $x, r=1, k=0.85, c_{3}=-0.084, A_{3}=1.41$. (d) Plot of the nondimensional intensity $\left|\phi_{4}\right|^{2}$ versus the nondimensional coordinate $x, r=1, k=0.85, c_{4}=-0.17, A_{4}=5.29$. (e) Plot of the nondimensional intensity $\left|\phi_{5}\right|^{2}$ versus the nondimensional coordinate $x, r=1, k=0.85, c_{5}=-0.030, A_{5}=4.54$. 


$$
\begin{aligned}
& \sum_{n=1}^{3}\left[2 \delta_{n}+\left(2 c_{n}-\delta_{n}^{2}\right) \sqrt{1-k^{2}}\right] A_{n}^{2}-\sum_{n=4}^{6}\left[2 c_{n}-\delta_{n}^{2}\right. \\
& \left.+2 \delta_{n} \sqrt{1-k^{2}}\right] A_{n}^{2}=0 \\
& \sum_{n=1}^{3}\left(\delta_{n}^{2}-2 c_{n}+2 c_{n} \delta_{n} \sqrt{1-k^{2}}\right) A_{n}^{2}-\sum_{n=4}^{6}\left[2 c_{n} \delta_{n}\right. \\
& \left.\quad+\left(\delta_{n}^{2}-2 c_{n}\right) \sqrt{1-k^{2}}\right] A_{n}^{2} \\
& =0, \\
& \sum_{n=1}^{3} c_{n}\left(2 \delta_{n}+c_{n} \sqrt{1-k^{2}}\right) A_{n}^{2}-\sum_{n=4}^{6} c_{n}\left(c_{n}+2 \delta_{n} \sqrt{1-k^{2}}\right) A_{n}^{2} \\
& =0, \quad \sum_{n=1}^{3} c_{n}^{2} A_{n}^{2}-\sqrt{1-k^{2}} \sum_{n=4}^{6} c_{n}^{2} A_{n}^{2}=42 r^{2} \sqrt{1-k^{2}} .
\end{aligned}
$$

Intensities of the components are illustrated in Figs. 2(a)2(f) for typical values of $k$. Up to six local maxima are possible for each period.

We verify by the software MATHEMATICA that the system Eqs. (5.1) and (5.2) satisfy Eq. (1.1).

\section{CNLS EQUATION FOR SIX COMPONENTS: ANOTHER SOLUTION}

A different combination of elliptic functions will generate another periodic solution for the CNLS system with six components. The "index of refraction" will attain a form different from the case in Sec. V. More precisely, for $n=1,2,3$ the components are ( $k$ is modulus of the Jacobi elliptic functions)

$$
\begin{aligned}
\phi_{n}= & A_{n}\left[c_{n}-\frac{\delta_{n} \mathrm{dn}^{2}(r x)}{\left(1-k^{2}\right)^{1 / 2}}-\frac{\mathrm{dn}^{4}(r x)}{1-k^{2}}\right] \\
& \times \frac{k \operatorname{sn}(r x) \operatorname{cn}(r x) \exp \left(-i \Omega_{n} t\right)}{\left(1-k^{2}\right)^{1 / 4}} .
\end{aligned}
$$

$c_{n}, n=1,2,3$ are roots of

$$
\begin{aligned}
& 363 c^{3}+\frac{11\left(37-37 k^{2}+8 k^{4}\right) c^{2}}{1-k^{2}}+\frac{\left(45-45 k^{2}+16 k^{4}\right) c}{1-k^{2}}+1 \\
& \quad=0
\end{aligned}
$$

$\delta_{n}, \Omega_{n}, n=1,2,3$ are related by

$$
\begin{gathered}
\delta_{n}=12\left(\sqrt{1-k^{2}}+\frac{1}{\sqrt{1-k^{2}}}\right)\left(\frac{1}{c_{n}}-11\right)^{-1}, \\
\Omega_{n}+h_{0}=-22 r^{2} \delta_{n} \sqrt{1-k^{2}}-25 r^{2}\left(2-k^{2}\right) .
\end{gathered}
$$

It is extremely important to note that $h_{0}$ in this case is different from that in Sec. V [Eq. (6.2) below].
For $n=4,5,6$ the remaining components are still given by Eqs. (5.2)-(5.5). The constant $h_{1}$ is still given by

$$
h_{1}=42 r^{2} \sqrt{1-k^{2}}
$$

but the constant $h_{0}$ is now,

$$
h_{0}=-\sum_{n=1}^{3} \frac{c_{n}^{2} \sqrt{1-k^{2}} A_{n}^{2}}{k^{2}} .
$$

The amplitude parameters $A_{n}, n=1, \ldots, 6$, are governed by

$$
\begin{aligned}
& \frac{\sqrt{1-k^{2}}}{k}\left(\sum_{n=1}^{3} A_{n}^{2}\right)-\left(\sum_{n=4}^{6} A_{n}^{2}\right)=0 \\
& \sum_{n=1}^{3} \frac{\left(2-k^{2}-2 \delta_{n} \sqrt{1-k^{2}}\right) A_{n}^{2}}{k}-\sum_{n=4}^{6}\left(\sqrt{1-k^{2}}-2 \delta_{n}\right) A_{n}^{2}=0 \\
& \sum_{n=1}^{3}\left[\frac{2 \delta_{n}\left(2-k^{2}\right)+\left(2 c_{n}-1-\delta_{n}^{2}\right) \sqrt{1-k^{2}}}{k}\right] A_{n}^{2} \\
& -\sum_{n=4}^{6}\left[2 c_{n}-\delta_{n}^{2}+2 \delta_{n} \sqrt{1-k^{2}}\right] A_{n}^{2} \\
& =0 \text {, } \\
& \sum_{n=1}^{3}\left[\frac{\left(\delta_{n}^{2}-2 c_{n}\right)\left(2-k^{2}\right)+2 \delta_{n}\left(c_{n}-1\right) \sqrt{1-k^{2}}}{k}\right] A_{n}^{2} \\
& -\sum_{n=4}^{6}\left(2 c_{n} \delta_{n}+\left(\delta_{n}^{2}-2 c_{n}\right) \sqrt{1-k^{2}}\right) A_{n}^{2}=0 \\
& \sum_{n=1}^{3}\left[\frac{2 c_{n} \delta_{n}\left(2-k^{2}\right)+\left(c_{n}^{2}+\delta_{n}^{2}-2 c_{n}\right) \sqrt{1-k^{2}}}{k}\right] A_{n}^{2} \\
& -\sum_{n=4}^{6} c_{n}\left(c_{n}+2 \delta_{n} \sqrt{1-k^{2}}\right) A_{n}^{2} \\
& =0 \text {, } \\
& \begin{array}{l}
\sum_{n=1}^{3} c_{n}\left[\frac{c_{n}\left(2-k^{2}\right)+2 \delta_{n} \sqrt{1-k^{2}}}{k}\right] A_{n}^{2}-\sum_{n=4}^{6} c_{n}^{2} \sqrt{1-k^{2}} A_{n}^{2} \\
\quad=42 r^{2} \sqrt{1-k^{2}}
\end{array}
\end{aligned}
$$

Plots for the intensities will be very similar to those from the preceding section, and hence will be omitted.

\section{CONCLUSIONS}

Periodic solutions for CNLS systems with five and six components are derived by a combination of the Hirota bilinear transformation, elliptic and theta functions. Systems of still higher order require an almost oppressive amount of algebra, and systematic use of symbolic manipulation package might be needed. The long wave limit is studied and- 


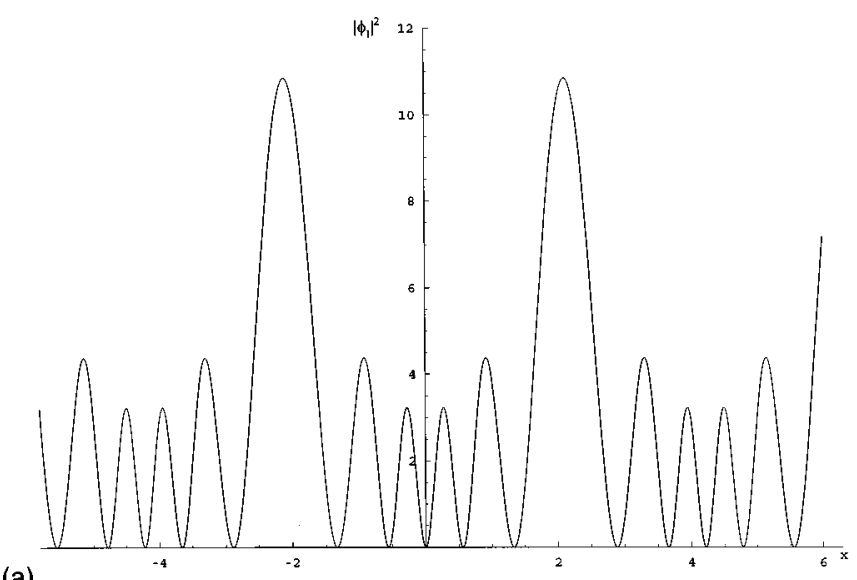

(a)

(b)
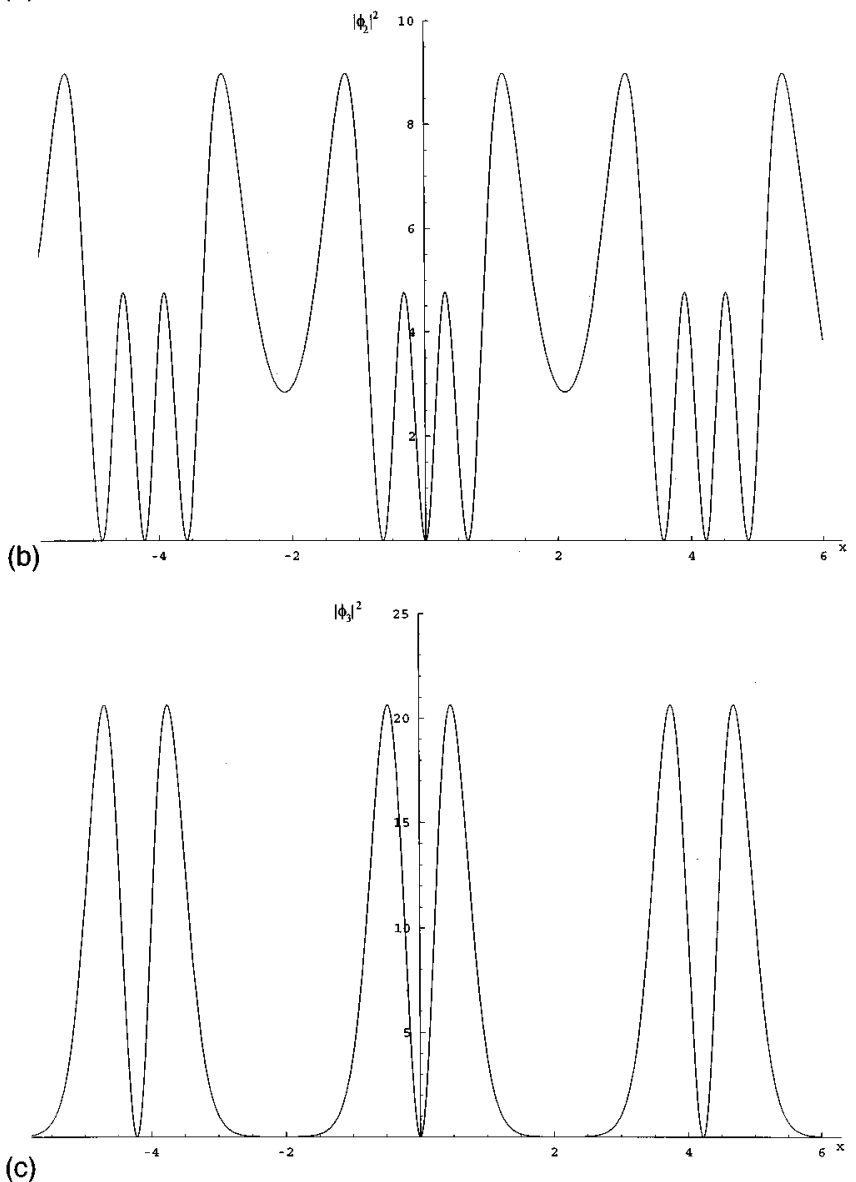

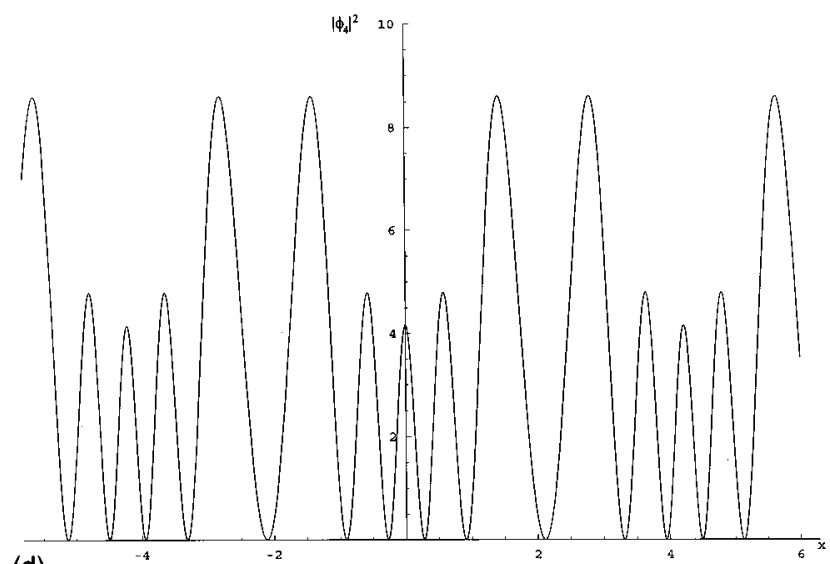

(d)

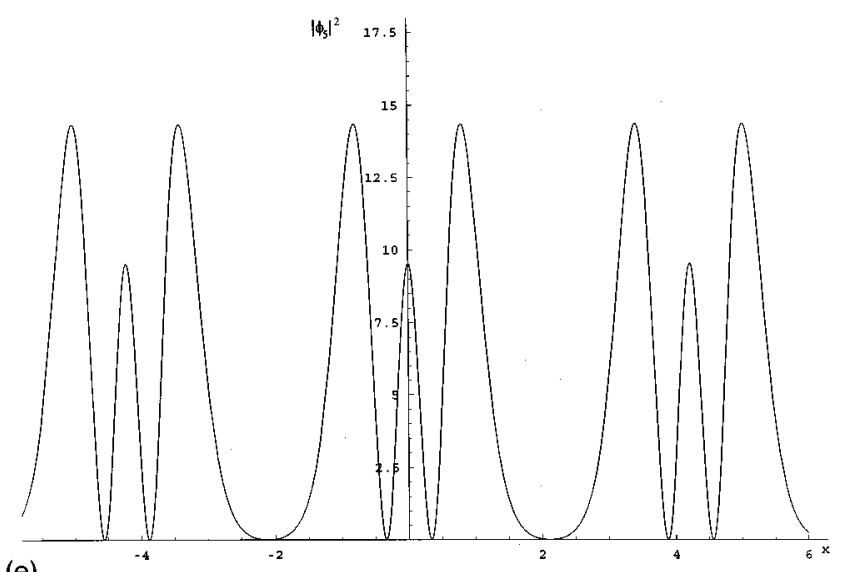

(e)

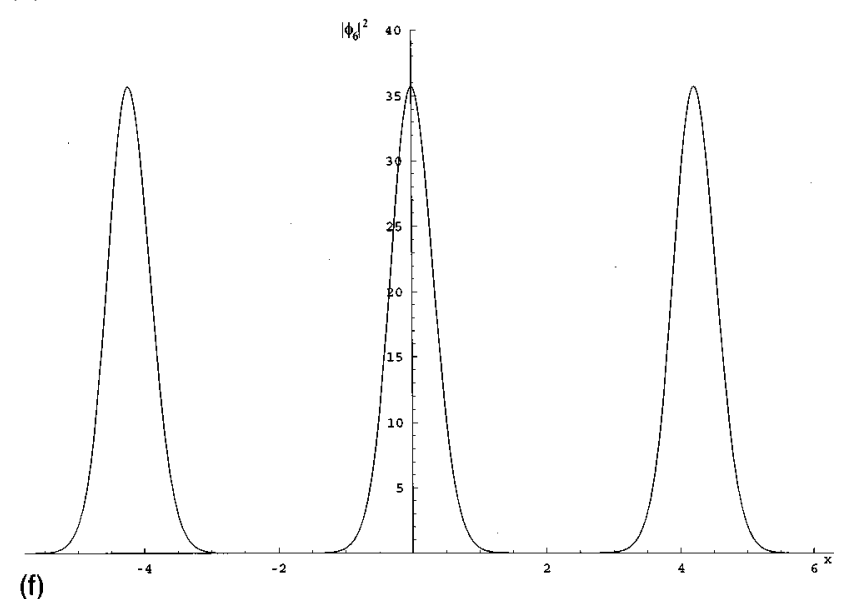

FIG. 2. (a) Plot of the nondimensional intensity $\left|\phi_{1}\right|^{2}$ versus the nondimensional coordinate $x, r=1, k=0.85, c_{1}=-1.19, A_{1}=19.83$. (b) Plot of the nondimensional intensity $\left|\phi_{2}\right|^{2}$ versus the nondimensional coordinate $x, r=1, k=0.85, c_{2}=-0.44, A_{2}=12.30$. (c) Plot of the nondimensional intensity $\left|\phi_{3}\right|^{2}$ versus the nondimensional coordinate $x, r=1, k=0.85, c_{3}=-0.79, A_{3}=5.15$. (d) Plot of the nondimensional intensity $\left|\phi_{4}\right|^{2}$ versus the nondimensional coordinate $x, r=1, k=0.85, c_{4}=-2.06, A_{4}=15.74$. (e) Plot of the nondimensional intensity $\left|\phi_{5}\right|^{2}$ versus the nondimensional coordinate $x, r=1, k=0.85, c_{5}=-0.40, A_{5}=7.17$. (f) Plot of the nondimensional intensity $\left|\phi_{6}\right|^{2}$ versus the nondimensional coordinate $x, r=1, k=0.85, c_{6}=-0.050, A_{6}=1.28$.

solitary waves solutions published in the literature are recovered. The precision and validity of the periodic solutions are verified independently by direct differentiation with a computer algebra software. Since results for CNLS systems of an arbitrary integer order are still scarce, the present work might provide some preliminary results in that direction. CNLS systems are useful in the description of partially coherent solitons in media with a slow Kerr-like nonlinearity. In general exact solutions will give a precise formulation of spatial beams. In particular, according to recent works on photorefractive crystals, the number of components in such CNLS systems can be large. Work and results for such higher order CNLS systems should prove to be of fundamental as well as practical interests. 


\section{ACKNOWLEDGMENT}

Partial financial support has been provided by the Hong Kong Research Grants Council Contracts Nos. HKU 7067/ 98E and HKU 7066/00E.

\section{APPENDIX}

The theta functions $\theta_{n}(x)[14,15], n=1,2,3,4$ and the parameters $q$ (the nome), $\tau$ (pure imaginary) are defined by

$$
\begin{gathered}
\theta_{1}(x)=\sum_{n=0}^{\infty}(-1)^{n} q^{(n+1 / 2)^{2}} \sin (2 n+1) x \\
=-\sum_{m=-\infty}^{\infty} \exp \left[\pi i \tau\left(m+\frac{1}{2}\right)^{2}+2 i\left(m+\frac{1}{2}\right)\left(x+\frac{\pi}{2}\right)\right], \\
\theta_{2}(x)=2 \sum_{n=0}^{\infty} q^{(n+1 / 2)^{2}} \cos (2 n+1) x \\
=\sum_{m=-\infty}^{\infty} \exp \left[\pi i \tau\left(m+\frac{1}{2}\right)^{2}+2 i\left(m+\frac{1}{2}\right) x\right], \\
\theta_{3}(x)=1+2 \sum_{n=1}^{\infty} q^{n^{2}} \cos 2 n x=\sum_{=-\infty}^{\infty} \exp \left(\pi i \tau m^{2}+2 i m x\right), \\
\theta_{4}(x)=1+2 \sum_{n=1}^{\infty}(-1)^{n} q^{n^{2}} \cos 2 n x \\
\quad \sum_{m=-\infty} \exp \left[\pi i \tau m^{2}+2 i m\left(x+\frac{\pi}{2}\right)\right],
\end{gathered}
$$

$K$ and $K^{\prime}$ are the complete elliptic integrals. Relationships between the theta and elliptic functions are

$$
\begin{gathered}
\operatorname{sn}(u)=\frac{\theta_{3}(0) \theta_{1}(z)}{\theta_{2}(0) \theta_{4}(z)}, \quad \operatorname{cn}(u)=\frac{\theta_{4}(0) \theta_{2}(z)}{\theta_{2}(0) \theta_{4}(z)}, \\
\operatorname{dn}(u)=\frac{\theta_{4}(0) \theta_{3}(z)}{\theta_{3}(0) \theta_{4}(z)}, \\
z=\frac{u}{\theta_{3}^{2}(0)}, \quad k=\frac{\theta_{2}^{2}(0)}{\theta_{3}^{2}(0)}, \quad k^{\prime}=\frac{\theta_{4}^{2}(0)}{\theta_{3}^{2}(0)}, \quad k^{2}+\left(k^{\prime}\right)^{2}=1 .
\end{gathered}
$$

Theta functions possess a huge variety of product identities, e.g.,

$$
\theta_{3}(x+y) \theta_{3}(x-y) \theta_{2}^{2}(0)=\theta_{4}^{2}(x) \theta_{1}^{2}(y)+\theta_{3}^{2}(x) \theta_{2}^{2}(y),
$$

$$
\theta_{4}(x+y) \theta_{4}(x-y) \theta_{2}^{2}(0)=\theta_{4}^{2}(x) \theta_{2}^{2}(y)+\theta_{3}^{2}(x) \theta_{1}^{2}(y) .
$$

Differentiating Eqs. (A7) and (A8) with respect to $y$ and setting $y=0$ yield

$$
\begin{aligned}
& D_{x}^{2} \theta_{3}(x) \cdot \theta_{3}(x)=\frac{2 \theta_{2}^{\prime \prime}(0) \theta_{3}^{2}(x)}{\theta_{2}(0)}+2 \theta_{3}^{2}(0) \theta_{4}^{2}(0) \theta_{4}^{2}(x), \\
& D_{x}^{2} \theta_{4}(x) \cdot \theta_{4}(x)=2 \theta_{3}^{2}(0) \theta_{4}^{2}(0) \theta_{3}^{2}(x)+\frac{2 \theta_{2}^{\prime \prime}(0) \theta_{4}^{2}(x)}{\theta_{2}(0)} .
\end{aligned}
$$

Hence formulas for $D_{x} \theta_{m} \cdot \theta_{n}, D_{x}^{2} \theta_{m} \cdot \theta_{n}$ can be developed for $m, n$ integers [16]. Derivatives for products of theta functions can be obtained by repeated use of identities such as

$$
\begin{gathered}
D_{x} a b \cdot c d=b d D_{x} a \cdot c+a c D_{x} b \cdot d, \\
D_{x}^{2} a b \cdot c d=b d D_{x}^{2} a \cdot c+a c D_{x}^{2} b \cdot d+2\left(D_{x} a \cdot c\right)\left(D_{x} b \cdot d\right) .
\end{gathered}
$$

Typical results for the purpose of the present discussion are

$$
\begin{aligned}
& D_{x}^{2} \theta_{4}^{5}(\alpha x) \cdot \theta_{4}^{5}(\alpha x)=5 \alpha^{2} \theta_{4}^{8}(\alpha x)\left(2 \theta_{3}^{2}(\theta) \theta_{4}^{2}(0) \theta_{3}^{2}(\alpha x)\right. \\
& \left.+\frac{2 \theta_{2}^{\prime \prime}(0) \theta_{4}^{2}(\alpha x)}{\theta_{2}(0)}\right) \\
& D_{x}^{2} \theta_{4}^{6}(\alpha x) \cdot \theta_{4}^{6}(\alpha x)=6 \alpha^{2} \theta_{4}^{10}(\alpha x)\left(2 \theta_{3}^{2}(0) \theta_{4}^{2}(0) \theta_{3}^{2}(\alpha x)\right. \\
& \left.+\frac{2 \theta_{2}^{\prime \prime}(0) \theta_{4}^{2}(\alpha x)}{\theta_{2}(0)}\right) \\
& D_{x}^{2} \theta_{1}(\alpha x) \theta_{2}(\alpha x) \theta_{3}^{3}(\alpha x) \cdot \theta_{4}^{5}(\alpha x) \\
& =\alpha^{2} \theta_{1}(\alpha x) \theta_{2}(\alpha x) \theta_{3}(\alpha x) \theta_{4}^{3}(\alpha x) \\
& \times\left[-20 \theta_{3}^{2}(0) \theta_{4}^{2}(0) \theta_{3}^{4}(\alpha x)+\left(\frac{2 \theta_{2}^{\prime \prime}(0)}{\theta_{2}(0)}+\frac{4 \theta_{3}^{\prime \prime}(0)}{\theta_{3}(0)}\right.\right. \\
& \left.+\frac{4 \theta_{4}^{\prime \prime}(0)}{\theta_{4}(0)}+12 \theta_{3}^{4}(0)+12 \theta_{4}^{4}(0)\right) \theta_{3}^{2}(\alpha x) \theta_{4}^{2}(\alpha x) \\
& \left.-6 \theta_{3}^{2}(0) \theta_{4}^{2}(0) \theta_{4}^{4}(\alpha x)\right] \\
& D_{x}^{2} \theta_{3}^{4}(\alpha x) \theta_{1}(\alpha x) \cdot \theta_{4}^{5}(\alpha x) \\
& =\alpha^{2} \theta_{1}(\alpha x) \theta_{3}^{2}(\alpha x) \theta_{4}^{3}(\alpha x)\left[-20 \theta_{3}^{2}(0) \theta_{4}^{2}(0) \theta_{3}^{4}(\alpha x)\right. \\
& +\left(\frac{\theta_{2}^{\prime \prime}(0)}{\theta_{2}(0)}+\frac{5 \theta_{3}^{\prime \prime}(0)}{\theta_{3}(0)}+\frac{4 \theta_{4}^{\prime \prime}(0)}{\theta_{4}(0)}+12 \theta_{3}^{4}(0)\right. \\
& \left.\left.+20 \theta_{4}^{4}(0)\right) \theta_{3}^{2}(\alpha x) \theta_{4}^{2}(\alpha x)-12 \theta_{3}^{2}(0) \theta_{4}^{2}(0) \theta_{4}^{4}(\alpha x)\right] \text {, }
\end{aligned}
$$




$$
\begin{aligned}
D_{x}^{2} \theta_{3}^{5}(\alpha x) \theta_{1}(\alpha x) \cdot \theta_{4}^{6}(\alpha x) \\
=\alpha^{2} \theta_{1}(\alpha x) \theta_{3}^{3}(\alpha x) \theta_{4}^{4}(\alpha x)\left[-30 \theta_{3}^{2}(0) \theta_{4}^{2}(\alpha x)\right. \\
\quad \times(0) \theta_{3}^{4}(\alpha x)+\left(\frac{\theta_{2}^{\prime \prime}(0)}{\theta_{2}(0)}+\frac{6 \theta_{3}^{\prime \prime}(0)}{\theta_{3}(0)}+\frac{5 \theta_{4}^{\prime \prime}(0)}{\theta_{4}(0)}\right.
\end{aligned}
$$

$$
\begin{aligned}
& +20 \theta_{3}^{4}(0)+30 \theta_{4}^{4}(0) \theta_{3}^{2}(\alpha x) \theta_{4}^{2}(\alpha x) \\
& -20 \theta_{3}^{2}(0) \theta_{4}^{2}(0) \theta_{4}^{4}(\alpha x)
\end{aligned}
$$

Similar results are obtained for the Hirota derivatives of other polynomials of theta functions of degree five or six, but details will be omitted here for brevity.
[1] M. Florjanczyk and R. Tremblay, Phys. Lett. A 141, 34 (1989).

[2] I. M. Uzunov, Opt. Commun. 83, 108 (1991).

[3] N. A. Kostov and I. M. Uzunov, Opt. Commun. 89, 389 (1992).

[4] M. Florjanczyk and R. Tremblay, Opt. Commun. 109, 405 (1994).

[5] F. T. Hioe, Phys. Rev. E 58, 6700 (1998).

[6] V. M. Petnikova, V. V. Shuvalov, and V. A. Vysloukh, Phys. Rev. E 60, 1009 (1999).

[7] F. T. Hioe, Phys. Rev. Lett. 82, 1152 (1999).

[8] F. T. Hioe, J. Phys. A 32, 1217 (1999).

[9] F. T. Hioe, J. Phys. A 32, 2415 (1999).

[10] K. Nakkeeran, Phys. Rev. E 62, 1313 (2000).
[11] D. E. Pelinovsky and Y. S. Kivshar, Phys. Rev. E 62, 8668 (2000).

[12] M. I. Carvalho, T. H. Coskun, D. N. Christodoulides, M. Mitchell, and M. Segev, Phys. Rev. E 59, 1193 (1999).

[13] N. Akhmediev and A. Ankiewicz, Phys. Rev. Lett. 82, 2661 (1999).

[14] Handbook of Mathematical Functions, edited by M. Abramowitz and I. Stegun (Dover, New York, 1965).

[15] D. F. Lawden, Elliptic Functions and Applications (SpringerVerlag, New York, 1989).

[16] K. W. Chow, J. Math. Phys. 36, 4125 (1995).

[17] K. W. Chow, Phys. Lett. A 285, 319 (2001).

[18] N. Akhmediev and A. Ankiewicz, Chaos 10, 600 (2000). 